J. Appl. Cryst. (1975). 8, 142

\title{
Local Arrangement of the Conducting Ions in the Super-Ionic Conductor $\beta_{\mathrm{Ag}}$-Alumina*
}

\author{
BY R. COMÈS
}

Laboratoire de Physique des Solides associé au CNRS, Bâtiment 510, Unicersité Paris-Sud, 91405 Orsay, France

\author{
And Y. Le Cars and J. Thery
}

Laboratoire de Chimie Appliquée de l'Etat Solide, 15 rue Georges Urbain, 94400 Vitry-sur-Seine, France

(Received 10 June 1974)

\begin{abstract}
The organization of the conducting ions in $\beta$-alumina is studied by means of $\mathrm{X}$-ray diffuse scattering as a function of temperature. While at high temperature $\left(750^{\circ} \mathrm{K}\right)$ the conducting ions are organized in a two-dimensional 'quasi-liquid' structure perpendicular to the $c$ axis, they form at low temperature $\left(77^{\circ} \mathrm{K}\right)$ microdomains corresponding to a two-dimensional superstructure with a lattice constant of $a \gamma^{\prime} 3=9.68 \AA$ ( $a=$ lattice constant of $\beta$-alumina $)$. The size of these microdomains which is about $50 \AA$ at $77^{\circ} \mathrm{K}$, decreases progressively with increasing temperature. These results give a qualitative explanation for the changes in the ionic conductivity which, from a low-temperature solid-like value, increases at high temperature and reaches values more compatible with a quasi-liquid state.
\end{abstract}

* Full paper published by Le Cars, Y., Comès, R., Deschamps, L. \& Thery, Y. [Acta Cryst. (1974). A 30, 305-309].

J. Appl. Cryst. (1975). 8, 142

\section{Theory of Diffuse Scattering from Crystals with Point Defects}

\author{
BY P. H. DEDERICHS
}

Institut für Festkörperforschung der Kernforschungsanlage, Jülich, Postfach 365, 517 Jülich, Germany (BRD)

(Received 29 April 1974)

The theory of the diffuse scattering from crystals with small concentrations of point defects and small clusters is reviewed [e.g. see Krivoglaz, M. A. (1969). Theory of X-Ray and Thermal Neutron Scattering by Real Crystals. New York: Plenum Press; Schmatz, W. (1975). X-Ray and Neutron Scattering Studies on Disordered Crystals, in: Treatise on Materials Science and Technology, Vol. 2. New York: Academic Press; Dederichs, P. H. (1973). J. Phys. F: Metal Phys. 3, 471]. The elastic scattered intensities of an ideal crystal are changed by defects in three different ways: (1) a shift of the Bragg intensities due to lattice expansion, (2) a reduction of the Bragg intensities by a static Debye-Waller factor, (3) the addition of a diffuse intensity in the whole Brillouin zone which will be discussed in detail. For small concentrations this diffuse intensity is proportional to the number of defects times the absolute square of a defect-structure factor which is determined by the defect itself and by the displaced lattice atoms in the vicinity of the defect. The displacements can be parametrized by Kanzaki forces which the defects exert on the neighbouring atoms. Between the Bragg reflexions the diffuse intensity is especially sensitive to the position of the defect and to the displacements of the nearby atoms, e.g., for interstitials, the calculated intensities for different positions are sufficiently different to allow a determination of the interstitial configurations. Near a Bragg reflexion the diffuse intensity is very strong and is determined by the long-range displacement field of the defect. This so-called Huang intensity varies radially as $1 / q^{2}$, whereas its angular variation reflects the symmetry of the dipole tensor. Depending on the defect symmetry and the reflexion choosen, three different types of isointensity surface can occur, thus allowing a determination of the defect symmetry from the topology of the isointensity surfaces. Besides the (symmetric) Huang intensity an asymmetry of the scattering near to the Bragg reflexion is observed, the sign of which depends on the sign of the displacement field around the defect. The diffuse intensity is strongly modified in the case of defect clustering. The Huang intensity very near to a Bragg reflexion increases in direct proportion to the number of point defects in the cluster, whereas for larger distances the intensity decreases as $1 / q^{4}$. 